\title{
Experimental study of the lifetime and phase transition in neutron-rich ${ }^{98,100,102} \mathrm{Zr}$
}

S. Ansari, ${ }^{1,2, *}$ J.-M. Régis, ${ }^{1}$ J. Jolie,,${ }^{1}$ N. Saed-Samii, ${ }^{1}$ N. Warr, ${ }^{1}$ W. Korten, ${ }^{2}$ M. Zielińska, ${ }^{2}$ M.-D. Salsac, ${ }^{2}$ A. Blanc, ${ }^{3}$ M. Jentschel, ${ }^{3}$ U. Köster, ${ }^{3}$ P. Mutti, ${ }^{3}$ T. Soldner, ${ }^{3}$ G. S. Simpson,${ }^{4}$ F. Drouet, ${ }^{4}$ A. Vancraeyenest,${ }^{4}$ G. de France,${ }^{5}$ E. Clément, ${ }^{5}$ O. Stezowski, ${ }^{6}$ C. A. Ur, ${ }^{7}$ W. Urban, ${ }^{8}$ P. H. Regan, ${ }^{9,10}$ Zs. Podolyák, ${ }^{9}$ C. Larijani, $,{ }^{9}, 10$ C. Townsley,${ }^{9}$ R. Carroll, ${ }^{9}$ E. Wilson, ${ }^{9}$ H. Mach, ${ }^{11, \dagger}$ L. M. Fraile, ${ }^{12}$ V. Paziy,,${ }^{12}$ B. Olaizola,${ }^{12,13}$ V. Vedia,${ }^{12}$ A. M. Bruce, ${ }^{14}$ O. J. Roberts, ${ }^{14}$ J. F. Smith ${ }^{15}$ M. Scheck,${ }^{15}$ T. Kröll, ${ }^{16}$ A.-L. Hartig, ${ }^{16}$ A. Ignatov, ${ }^{16}$ S. Ilieva, ${ }^{16}$ S. Lalkovski, ${ }^{17}$ N. Mărginean, ${ }^{18}$ T. Otsuka, ${ }^{19,20,21,22,23}$ N. Shimizu, ${ }^{20}$ T. Togashi, ${ }^{20}$ and Y. Tsunoda ${ }^{20}$

${ }^{1}$ Institut für Kernphysik der Universität zu Köln, Zülpicher Str. 77, D-50937 Köln, Germany

${ }^{2}$ Irfu, CEA, Université Paris-Saclay, F-91191 Gif-sur-Yvette, France

${ }^{3}$ Institut Laue-Langevin, 71 Avenue des Martyrs, F-38042 Grenoble Cedex, France

${ }^{4}$ LPSC, 53 Avenue des Martyrs, F-38026 Grenoble Cedex, France

${ }^{5}$ Grand Accélérateur National d'Ions Lourds (GANIL), CEA/CRF-CNRS/IN2P3, F-14076 Caen Cedex 05, France

${ }^{6} I P N$ de Lyon, 4, Rue Enrico Fermi, F-69622 Villeurbanne Cedex, France

${ }^{7}$ INFN, via Marzolo 8, I-35131 Padova, Italy

${ }^{8}$ Faculty of Physics, University of Warsaw, ul. Pasteura 5, PL-02-093 Warsaw, Poland

${ }^{9}$ Department of Physics, University of Surrey, Guildford GU2 7XH, United Kingdom

${ }^{10}$ National Physical Laboratory, Teddington, Middlesex, TW11 OLW, United Kingdom

${ }^{11}$ National Centre for Nuclear Research, Andrzeja Sottana 7, PL-05-400 Otwock-Swierk, Poland

${ }^{12}$ Grupo de Fisica Nuclear, FAMN, Universidad Complutense, E-28040 Madrid, Spain

${ }^{13}$ TRIUMF, 4004 Wesbrook Mall, Vancouver, British Columbia, V6T 2A3, Canada

${ }^{14}$ School of Computing, Engineering and Mathematics, University of Brighton, Brighton, BN2 4GJ, United Kingdom

${ }^{15}$ School of Engineering and Computing, University of the West of Scotland, High Street, Paisley, PA1 2BE, United Kingdom

${ }^{16}$ Institut für Kernphysik, TU Darmstadt, Schlossgartenstr. 7, D-64289 Darmstadt, Germany

${ }^{17}$ Faculty of Physics, University of Sofia, BG-1164 Sofia, Bulgaria

${ }^{18}$ Horia Hulubei NIPNE, R-77125 Bucharest, Romania

${ }^{19}$ RIKEN Nishina Center, 2-1 Hirosawa, Wako, Saitama 351-0198, Japan

${ }^{20}$ Center for Nuclear Study, University of Tokyo, Hongo, Bunkyo-ku Tokyo 113-0033, Japan

${ }^{21}$ Department of Physics, University of Tokyo, Hongo, Bunkyo-ku Tokyo 113-0033, Japan

${ }^{22}$ NSCL, Michigan State University, East Lansing, Michigan 48824, USA

${ }^{23}$ Instituut voor Kern- en Stralingsfysica, KU Leuven, B-3001 Leuven, Belgium

(Received 19 July 2017; published 27 November 2017)

\begin{abstract}
Rapid shape changes are observed for neutron-rich nuclei with $A$ around 100. In particular, a sudden onset of ground-state deformation is observed in the $\mathrm{Zr}$ and $\mathrm{Sr}$ isotopic chains at $N=60$ : Low-lying states in $N \leqslant 58$ nuclei are nearly spherical, while those with $N \geqslant 60$ have a rotational character. Nuclear lifetimes as short as a few picoseconds can be measured using fast-timing techniques with $\mathrm{LaBr}_{3}(\mathrm{Ce})$ scintillators, yielding a key ingredient in the systematic study of the shape evolution in this region. We used neutron-induced fission of ${ }^{241} \mathrm{Pu}$ and ${ }^{235} \mathrm{U}$ to study lifetimes of excited states in fission fragments in the $A \sim 100$ region with the EXILL-FATIMA array located at the PF1B cold neutron beam line at the Institut Laue-Langevin. In particular, we applied the generalized centroid difference method to deduce lifetimes of low-lying states for the nuclei ${ }^{98} \mathrm{Zr}(N=58),{ }^{100} \mathrm{Zr}$, and ${ }^{102} \mathrm{Zr}(N \geqslant 60)$. The results are discussed in the context of the presumed phase transition in the $\mathrm{Zr}$ chain by comparing the experimental transition strengths with the theoretical calculations using the interacting boson model and the Monte Carlo shell model.
\end{abstract}

DOI: 10.1103/PhysRevC.96.054323

\section{INTRODUCTION}

The last few decades have seen a focus on the shape-phase transition in nuclei around $A=100$. The appearance of strong quadrupole deformation beyond $N=60$ in the $A \sim 100$ mass region was discovered in the 1960s by Johansson [1] in a study of $\gamma$ rays emitted by fission fragments. Soon after, Cheifetz etal. [2] observed regular rotational bands

\footnotetext{
*saba.ansari@cea.fr

${ }^{\dagger}$ Deceased
}

in neutron-rich $\mathrm{Zr}, \mathrm{Mo}, \mathrm{Ru}$, and $\mathrm{Pd}$ isotopes populated in spontaneous fission of ${ }^{252} \mathrm{Cf}$. In particular, the lifetimes of the $2_{1}^{+}$states in ${ }^{100,102} \mathrm{Zr}$ obtained in that study [2] confirmed their highly deformed character. These experimental discoveries triggered an important theoretical effort to explain the origin of quadrupole deformation in $A \sim 100$ nuclei; early calculations are described, for example, in Refs. [3,4].

The simplest estimate of nuclear deformation can be obtained from the energy of the $2_{1}^{+}$state in even-even nuclei. For $\operatorname{Sr}(Z=38)$ and $\operatorname{Zr}(Z=40)$ isotopes it is observed to decrease dramatically at $N=60$, while the evolution is much more gradual in Mo nuclei $(Z=42)$ (see Fig. 1). A gradual decrease 


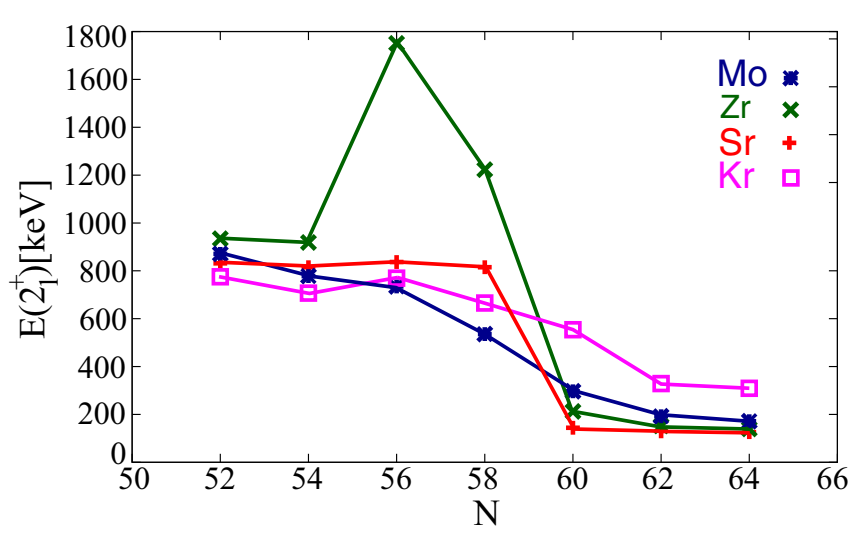

FIG. 1. Evolution of the $2_{1}^{+}$excitation energy as a function of neutron number in the $A \sim 100$ region. The transition energies are taken from National Nuclear Data Center [7] and the recent results for ${ }^{98,100} \mathrm{Kr}$ are adopted from Ref. [6].

of the $2_{1}^{+}$energy is also observed for ${ }^{92,94,96} \mathrm{Kr}$ nuclei $(Z=36)$. This is consistent with the results of mass measurements for ${ }^{96,97} \mathrm{Kr}[5]$ that show a smooth evolution towards the dripline in contrast to the sharp changes observed for heavier $N=60$ nuclei. However, a significant drop in energy was observed for the $2_{1}^{+}$state in ${ }^{98} \mathrm{Kr}$ [6]. This energy further stabilized at ${ }^{100} \mathrm{Kr}$ [6], which suggests that a shape transition may appear in the $\mathrm{Kr}$ isotopic chain at $N=62$ instead of $N=60$. Judging by level energies alone, the $Z$ boundaries of the region of the shape transition at $N=60$ seem to be clearly defined.

The $R_{4 / 2}=E\left(4_{1}^{+}\right) / E\left(2_{1}^{+}\right)$ratios for $N \geqslant 60 \mathrm{Sr}, \mathrm{Zr}$, Mo, and $\mathrm{Ru}$ nuclei have a value around 3 [2], which is expected for a rigid rotor and is consistent with a static character of the deformation in this mass region. Again, a very different behavior was recently observed in ${ }^{96} \mathrm{Kr}$, with the $R_{4 / 2}$ value dropping abruptly to 2.1 , suggesting a dynamical character of the deformation [8].

A similar picture is emerging from measurements of transition probabilities. A Coulomb excitation study of ${ }^{96} \mathrm{Kr}$ [9] yielded a $B\left(E 2 ; 2_{1}^{+} \rightarrow 0_{1}^{+}\right)$value much lower than those for ${ }^{98} \mathrm{Sr}$ and ${ }^{100} \mathrm{Zr}$, and only slightly higher than that for ${ }^{94} \mathrm{Kr}$ [9]. In contrast, regular rotational ground-state bands were observed in ${ }^{97,99} \mathrm{Rb}$ [10], and the obtained transition probabilities show that the deformation of these nuclei is essentially the same as that observed inside the well-deformed region, thus establishing ${ }^{97} \mathrm{Rb}$ as its cornerstone. Recent lifetime measurements for ${ }^{99,101} \mathrm{Y}$ and ${ }^{101,103,105} \mathrm{Nb}$ [11] confirmed that these nuclei are as deformed as the neighboring even-even isotopes with $N \geqslant 60$.

Sudden shape changes may be interpreted as a result of an inversion of two distinct configurations associated with different nuclear shapes. Indeed, the shape transition at $N=60$ is accompanied by the appearance of low-lying $0_{2}^{+}$states indicating possible shape coexistence [12] and, similar to the $2_{1}^{+}$state, an abrupt drop of the $0_{2}^{+}$energy is observed at $N=60$. The recent Coulomb excitation study of ${ }^{96,98} \mathrm{Sr}[13,14]$ provided firm evidence for configuration inversion in these nuclei, demonstrating important similarities in terms of transition probabilities and spectroscopic quadrupole moments between the ground-state band in ${ }^{96} \mathrm{Sr}$ and the structure built on the $\mathrm{O}_{2}^{+}$state in ${ }^{98} \mathrm{Sr}$. These conclusions are consistent with the results of a new lifetime measurement in the $\mathrm{Sr}$ isotopic chain [15]. The interpretation of $E 2$ matrix elements obtained in the Coulomb excitation measurement $[13,14]$ using the two-state mixing model points to very low mixing between prolate and spherical configurations in the wave functions of the $0_{1}^{+}$states in ${ }^{98} \mathrm{Sr}$, in spite of their proximity in energy. The same conclusion can be drawn from the measured $E 0$ transition strength between the $0_{2}^{+}$and the $0_{1}^{+}$states in ${ }^{98} \mathrm{Sr}[16,17]$ and also from $E 0$ and $E 2$ transition strengths in ${ }^{100} \mathrm{Zr}$ [18-20]. The weak mixing of the coexisting structures in ${ }^{98} \mathrm{Sr}$ and ${ }^{100} \mathrm{Zr}$ is very different from that observed for other regions of shape coexistence, for example, in ${ }^{74,76} \mathrm{Kr}$ [21] and ${ }^{182-188} \mathrm{Hg}$ [22] isotopes, where strong mixing makes the change of the ground-state properties more gradual.

The local character of the shape change suggests that specific proton and neutron orbitals are responsible for this effect. Unfortunately, the valence space required to describe $A \sim 100$ nuclei is currently too large for conventional shell model calculations, although they could correctly describe the properties of the light $(N<60) \mathrm{Zr}$ isotopes [23]. However, recent advances with the Monte Carlo shell model have made it possible to investigate the origin of the shape transition at $N=60$ [24] and relate it to the strong proton-neutron interaction between proton $\pi 1 g_{9 / 2}$ and neutron $\nu 1 g_{7 / 2}$ subshells. Promotion of protons from the $\pi 2 p_{1 / 2}$ to the $\pi 1 g_{9 / 2}$ orbital causes the reduction in the spin-orbit coupling for neutron orbitals, reducing the $\nu 2 d_{5 / 2}-v 1 g_{7 / 2}$ gap. Increased occupation of the $v 1 g_{7 / 2}$ orbital leads in turn to an increase in spin-orbit splitting in the proton sector and reduction of the $\pi 2 p_{1 / 2}-\pi 1 g_{9 / 2}$ gap. This self-reinforcing effect, known as type-II shell evolution [25], is suggested to be responsible for the appearance of deformed states in $\mathrm{Zr}$ isotopes. Because these specific particle-hole excitations lead to a significant reorganization of the effective single-particle energies, the mixing of normal states and those with deformation-optimized shell structure is suppressed, consistent with experimental results. The calculations of Togashi et al. [24] predict a dramatic shape change between the ground states of ${ }^{98} \mathrm{Zr}$ and ${ }^{100} \mathrm{Zr}$, with the $\mathrm{O}_{2}^{+}$in ${ }^{98} \mathrm{Zr}$ becoming the $0_{1}^{+}$state of ${ }^{100} \mathrm{Zr}$ and the ground state of ${ }^{98} \mathrm{Zr}$ becoming the nonyrast $0_{2}^{+}$state in ${ }^{100} \mathrm{Zr}$ and beyond.

The current paper presents new experimental results on lifetimes in neutron-rich $\mathrm{Zr}$ isotopes, which bring systematic information on the evolution of nuclear deformation and collectivity in the vicinity of the $N=60$ shape transition. The measured transition strengths are compared to the results of Monte Carlo shell model and IBM-1 calculations to get a better understanding of the shape transition and configuration inversion in the $\mathrm{Zr}$ isotopic chain.

\section{EXPERIMENT}

Lifetimes of low-lying excited states of ${ }^{98,100,102} \mathrm{Zr}$ have been measured through a prompt-fission spectroscopy experiment performed at the Institut Laue-Langevin (ILL) Grenoble, France. In this experiment, the high-flux cold neutron beam at PF1B [26] was inducing the fission reactions on targets of 
${ }^{235} \mathrm{U}$ and ${ }^{241} \mathrm{Pu}$. The EXILL-FATIMA setup consisted of eight EXOGAM clovers and $16 \mathrm{LaBr}_{3}(\mathrm{Ce})$ detectors, which were placed at a distance of $14.5 \mathrm{~cm}$ and $8.5 \mathrm{~cm}$, respectively, from the target [27]. Each target was sandwiched between Be layers to stop the fission fragments. The $\mathrm{LaBr}_{3}(\mathrm{Ce})$ detectors were arranged in a compact configuration to maximize the number of $\gamma-\gamma$ coincidences. A detailed description of the collimation of the neutron beam can be found in Ref. [28], the detector arrangement and analog fast-timing electronics in Ref. [27], and the triggerless data acquisition system in Ref. [29].

\section{A. Data analysis}

The data were sorted using a C++-based software, SOCOV2 [30], developed at the Institute of Nuclear Physics, Cologne. For the present application, coincidences between exactly one clover (after add-back) and two $\mathrm{LaBr}_{3}(\mathrm{Ce})$ detectors were required within the 120-ns time window, meaning the $\gamma$-ray multiplicity was equal to three.

The modern fast-timing method of mirror symmetric centroid difference (MSCD) [31] was used in the present work for lifetime determination. By using the feeding (decay) transition of a sequential $\gamma-\gamma$ cascade as the start signal of a time-to-amplitude converter (TAC) module and the decay (feeder) transition as the stop, we observed a signal delayed (antidelayed) by the lifetime $\tau$ of the decaying state. The centroid of the resulting TAC spectrum is thus shifted by $\tau$ (respectively, $-\tau$ ) from its prompt position. The MSCD method is based on the difference between the centroids of these two independent time distributions of a sequential $\gamma-\gamma$ cascade. This method considers the centroid difference as a physical observable and as the name suggests, interprets the centroid difference of the $\gamma-\gamma$ cascade as mirror symmetric with respect to a start-stop inversion, or equivalently, to a hypothetical inversion of the transitions in the cascade. The MSCD method in the case of no background is described by the following equation:

$$
\begin{aligned}
& \triangle C \\
& \left.\quad=E_{\text {feeder }}, E_{\text {decay }}\right) \\
& \quad=C_{\text {delayed }}-C_{\text {antidelayed }}\left(E_{\text {feeder }}, E_{\text {decay }}\right)-C^{A D}\left(E_{\text {decay }}, E_{\text {feeder }}\right) \\
& =\operatorname{PRD}\left(E_{\text {feeder }}, E_{\text {decay }}\right)+2 \tau,
\end{aligned}
$$

where $\mathrm{C}^{D}$ describes the centroid of the delayed time distribution and $\mathrm{C}^{\mathrm{AD}}$ is the centroid of the antidelayed one. The PRD is the prompt response difference which describes the combined $\gamma-\gamma$ time walk of the setup. The PRD for two $\gamma$-ray energies in a $\gamma-\gamma$ cascade is given as

$$
\operatorname{PRD}\left(E_{\text {feeder }}, E_{\text {decay }}\right)=\operatorname{PRD}\left(E_{\text {feeder }}\right)-\operatorname{PRD}\left(E_{\text {decay }}\right),
$$

and

$$
\begin{aligned}
\operatorname{PRD}\left(E_{\text {feeder }}, E_{\text {decay }}\right) & =\operatorname{PRD}_{E_{\text {decay }}}\left(E_{\text {feeder }}\right) \\
& =-\operatorname{PRD}_{E_{\text {feeder }}}\left(E_{\text {decay }}\right),
\end{aligned}
$$

where $\operatorname{PRD}_{E_{\text {decay }}}\left(E_{\text {feeder }}\right)$ [respectively, $\left.\operatorname{PRD}_{E_{\text {feeder }}}\left(E_{\text {decay }}\right)\right]$ is the prompt response difference at the energy of the feeding (respectively, decay) transition when the reference energy is at the decay (respectively, feeding) transition. This shows

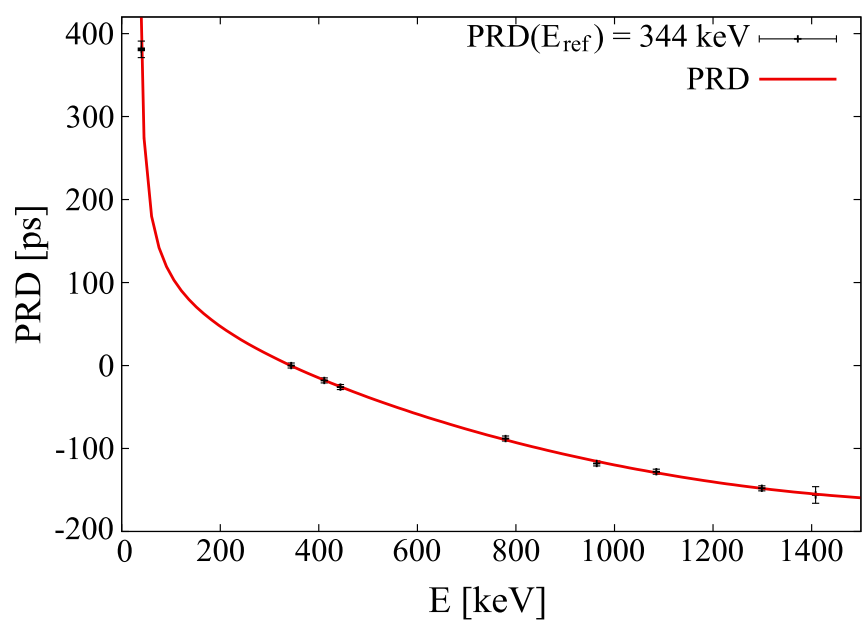

FIG. 2. PRD curve obtained using a ${ }^{152} \mathrm{Eu}$ source.

the mirror symmetry of the method in which both PRD and centroid difference are mirror symmetric.

The PRD calibration is performed using a standard ${ }^{152} \mathrm{Eu}$ source. The calculated PRD was fitted by using the following calibration equation:

$$
\operatorname{PRD}\left(E_{\gamma}\right)=\frac{a}{\sqrt{b+E_{\gamma}}}+c \cdot E_{\gamma}+d \cdot E_{\gamma}^{2}+e,
$$

where $a, b, c, d$, and $e$ are the fit parameters. In the present case the PRD curve is adjusted for $E_{\text {ref }}=344 \mathrm{keV}$ (i.e., the value of the PRD at $344 \mathrm{keV}$ is $0 \mathrm{ps}$ ). The uncertainty on the PRD, $\delta(\mathrm{PRD})$, was obtained from the fit residual (mean root squared derivation) and is equal to $10 \mathrm{ps}$ within the $3 \sigma$ limit. The PRD curve (shown in Fig. 2) can be used to read the PRD value for any sequential $\gamma-\gamma$ cascade within the energy range of $0-1400 \mathrm{keV}$. The anode pulse was used for timing because it provides a stable, count-rate independent signal which results in a constant time-walk characteristic, i.e., the shape of the PRD curve does not change. This was explored and verified from data collected over five weeks using different $\gamma$-ray sources and $(\mathrm{n}, \gamma)$ reactions with detector count rates ranging from 3 to $25 \mathrm{kHz}$ [27]. The determination of the timing uncertainties and the PRD calibration procedure of the EXILLFATIMA setup are described in detail in [27].

The MSCD method was extended to the generalized centroid difference method (GCDM) for the system of $N$ nearly identical fast-timing detectors, as in the case of the EXILL-FATIMA campaign. In this method, instead of evaluating individual centroid differences for " $C_{\text {start, stop }}$ " between two independent timing distributions, the superimposed TAC spectrum of all the combinations of "start, stop" belonging to the $N$-detector system is evaluated [32]. Similar to Eq. (1), the relation between the mean centroid difference $\left[\overline{\triangle C\left(E_{\gamma}\right)}\right]$ and the mean prompt response difference $(\overline{\mathrm{PRD}})$ is given by

$$
\overline{\triangle C_{\mathrm{FEP}}}=\overline{\mathrm{PRD}}+2 \tau,
$$

where FEP stands for full-energy peak. Equation (5) is valid if the time differences between the start and the stop events are statistically distributed around the mean $\overline{\triangle C_{\mathrm{FEP}}}$ or $\overline{\mathrm{PRD}}$, and are independent of the detector-detector combination. 


\section{B. Lifetime determination}

We have measured the lifetimes of the $2_{1}^{+}$and $4_{1}^{+}$states of ${ }^{98} \mathrm{Zr}$ and $2_{1}^{+}, 4_{1}^{+}$and $6_{1}^{+}$states of ${ }^{100,102} \mathrm{Zr}$, analyzing the data collected with each of the targets $\left({ }^{235} \mathrm{U}\right.$ and $\left.{ }^{241} \mathrm{Pu}\right)$ separately. We present the details of the analysis procedure using the examples of the $2_{1}^{+}$and $4_{1}^{+}$states of ${ }^{100,102} \mathrm{Zr}$.

The most prominent source of background in the lowenergy range $(\leqslant 300 \mathrm{keV})$ for the EXILL-FATIMA setup was the Compton scattering. It arises from the superposition of Compton continua of multiple $\gamma$ rays produced in the fission process. In an ideal setup, Eq. (5) can be used for lifetime determination, however, in a real setup the experimental centroid difference ( $\triangle C_{\text {exp }}$ ) must be corrected to account for the Compton background $\left(\triangle C_{\mathrm{BG}}\right)$, following:

$$
\Delta C_{\mathrm{FEP}}=\Delta C_{\mathrm{exp}}+\frac{\Delta C_{\exp }-\Delta C_{\mathrm{BG}}}{p / b},
$$

where $p / b$ is the peak to background ratio. Equation (6) can be used for the Compton background correction when only one background component is present [27,31]. However, because two FEPs (feeder and decay) are used in the lifetime analysis, the Compton background underneath each of the FEPs in the $\gamma-\gamma$ cascade must be considered separately [15]:

$$
\triangle C_{\mathrm{FEP}}=\Delta C_{\exp }+\frac{1}{2}\left[t_{\text {corr }}(\text { feeder })+t_{\text {corr }}(\text { decay })\right],
$$

where

$$
\begin{aligned}
& t_{\text {corr }}(\text { feeder })=\left[\frac{\left(\triangle C_{\text {exp }}-\Delta C_{\mathrm{BG}}\right)}{p / b}\right]_{\text {feeder }}, \\
& t_{\text {corr }}(\text { decay })=\left[\frac{\left(\triangle C_{\text {exp }}-\Delta C_{\mathrm{BG}}\right)}{p / b}\right]_{\text {decay }},
\end{aligned}
$$

and

$$
\tau=\frac{1}{2}\left(\triangle C_{\mathrm{FEP}}-\mathrm{PRD}\right) .
$$

In Eqs. (7) and (9), $\triangle C_{\text {exp }}$ is the experimental value, $\triangle C_{\mathrm{FEP}}$ is the one related to FEP events only, corrected for the contribution of the Compton background $\left(\triangle C_{\mathrm{BG}}\right)$. The term $t_{\text {corr }}$ (feeder) [resp ectively, $t_{\text {corr }}$ (decay)] in Eq. (8) is the background correction resulting from the feeding (decay) transition in a spectrum gated on the decay (feeding) transition, and hence at the reference energy $\left(E_{\text {ref }}\right)$. When estimating the uncertainty on the lifetime, the individual contributions are taken into account as follows:

$$
\delta \tau=\frac{1}{2} \sqrt{\delta \Delta C_{\exp }^{2}+\delta t_{\text {corr }}^{2}+\delta \mathrm{PRD}^{2}},
$$

where $\delta t_{\text {corr }}$ corresponds to the mean uncertainty of the two Compton background correction terms.

The high multiplicity of $\gamma$ rays produced in the fission process can sometimes lead to erroneous results. For example, the transitions of interest (feeder and decay) for the lifetime measurements of $4_{1}^{+}$and $6_{1}^{+}$states of ${ }^{100,102} \mathrm{Zr}$ lie in the same energy range (480-500 keV) as the low-lying $\gamma$-ray transitions in ${ }^{138} \mathrm{Xe} \cdot{ }^{138} \mathrm{Xe}$ is one of the possible complementary partners of both ${ }^{100} \mathrm{Zr}$ and ${ }^{102} \mathrm{Zr}$ in the ${ }^{241} \mathrm{Pu}$ fission, through

$$
\begin{aligned}
{ }_{94}^{241} \mathrm{Pu}+n_{\mathrm{th}} \rightarrow & { }_{40}^{102} \mathrm{Zr}_{62}+{ }_{54}^{138} \mathrm{Xe}, 2 n \text { emission, } \\
& { }_{40}^{100} \mathrm{Zr}_{62}+{ }_{54}^{138} \mathrm{Xe}, 4 n \text { emission. }
\end{aligned}
$$

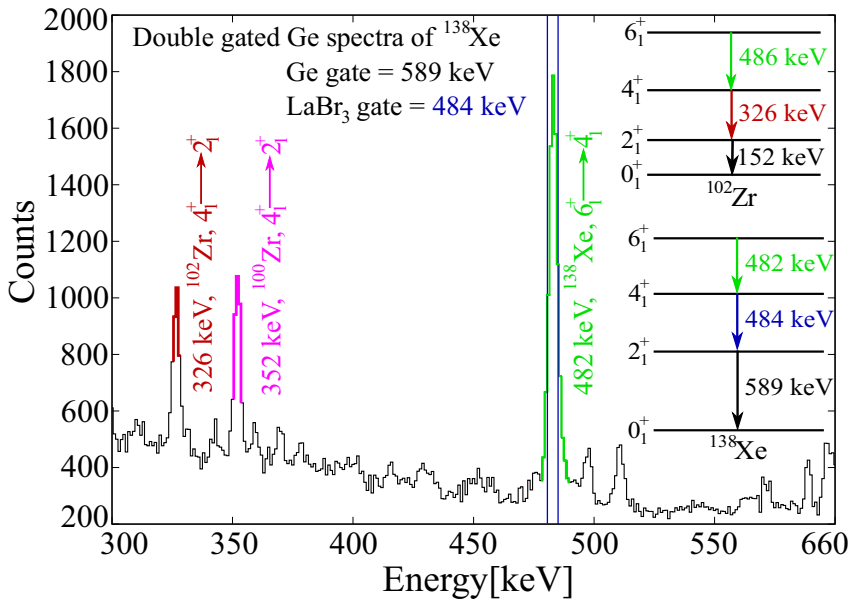

FIG. 3. The double-gated spectrum of ${ }^{138} \mathrm{Xe}$ which is a complementary partner of ${ }^{100} \mathrm{Zr}$ and ${ }^{102} \mathrm{Zr}$. Prominent peaks in the spectrum (shown in different colors) correspond to transitions in ${ }^{100} \mathrm{Zr},{ }^{102} \mathrm{Zr}$, and ${ }^{138} \mathrm{Xe}$.

This is illustrated by Fig. 3 showing a double-gated $(\mathrm{Ge}+$ $\left.\mathrm{LaBr}_{3}\right)$ spectrum of ${ }^{241} \mathrm{Pu}$ fission products, gated on two transitions in ${ }^{138} \mathrm{Xe}: 2_{1}^{+} \rightarrow 0_{1}^{+}(589 \mathrm{keV})$ observed with the $\mathrm{Ge}$ detectors and $4_{1}^{+} \rightarrow 2_{1}^{+}(484 \mathrm{keV})$ with $\mathrm{LaBr}_{3}(\mathrm{Ce})$ detectors. In addition to prominent $\gamma$ rays in ${ }^{138} \mathrm{Xe}$, one can also see $\gamma$ rays (highlighted in Fig. 3) originating from ${ }^{100,102} \mathrm{Zr}$, proving that ${ }^{138} \mathrm{Xe}$ and ${ }^{100,102} \mathrm{Zr}$ are complementary partners.
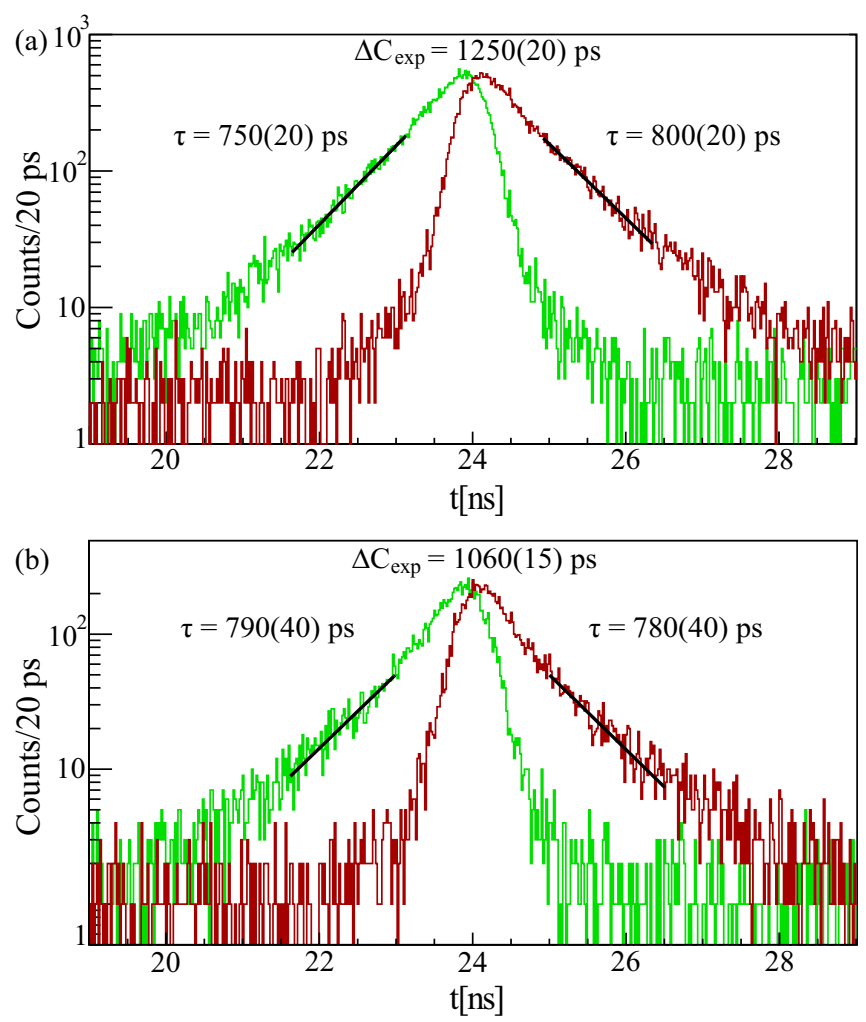

FIG. 4. Lifetime determination using the slope method for the $2_{1}^{+}$state of ${ }^{100} \mathrm{Zr}$. (a) Displays the delayed (red) and the antidelayed [green (light gray)] time distributions of the state of interest from ${ }^{235} \mathrm{U}$ fission, and (b) from ${ }^{241} \mathrm{Pu}$ fission. 
As these nuclei happen to have transitions with almost identical energies, the time spectra gated on the $486 \mathrm{keV}$ from ${ }^{102} \mathrm{Zr}$ and $497 \mathrm{keV}$ from ${ }^{100} \mathrm{Zr}$ will be contaminated by the $482 \mathrm{keV}$ and $484 \mathrm{keV}$ from ${ }^{138} \mathrm{Xe}$, resulting in biased lifetimes. This is, however, not the case for the fission of ${ }^{235} \mathrm{U}$, where Te nuclei are fission partners of ${ }^{100,102} \mathrm{Zr}$, and thus the lifetimes of the $4_{1}^{+}$and $6_{1}^{+}$states of ${ }^{100,102} \mathrm{Zr}$ can be correctly determined from the latter data set.

\section{1. ${ }^{98} \mathrm{Zr}$}

Level lifetimes in ${ }^{98} \mathrm{Zr}$ were investigated for the $2_{1}^{+}$and $4_{1}^{+}$ states. The lifetime analysis for the $2_{1}^{+}$state is done by using the $1223-\mathrm{keV} 2_{1}^{+} \rightarrow 0_{1}^{+}$transition depopulating this state as a stop and that feeding it $\left(621 \mathrm{keV}, 4_{1}^{+} \rightarrow 2_{1}^{+}\right)$as a start. The latter is used as the reference for the PRD. In addition, a gate on Ge singles is applied on the $6_{1}^{+} \rightarrow 4_{1}^{+}$transition at $647 \mathrm{keV}$ to select the cascade of interest and to improve the peak-tobackground ratio. The correction for the Compton background that lies underneath the peak of interest is applied using Eq. (7). Because of the uncertainties in PRD and Compton background correction for both fission targets, the lifetime of the shortlived $2_{1}^{+}$level of ${ }^{98} \mathrm{Zr}$ could not be determined precisely and only an upper limit is obtained. The feeding (647 keV) and
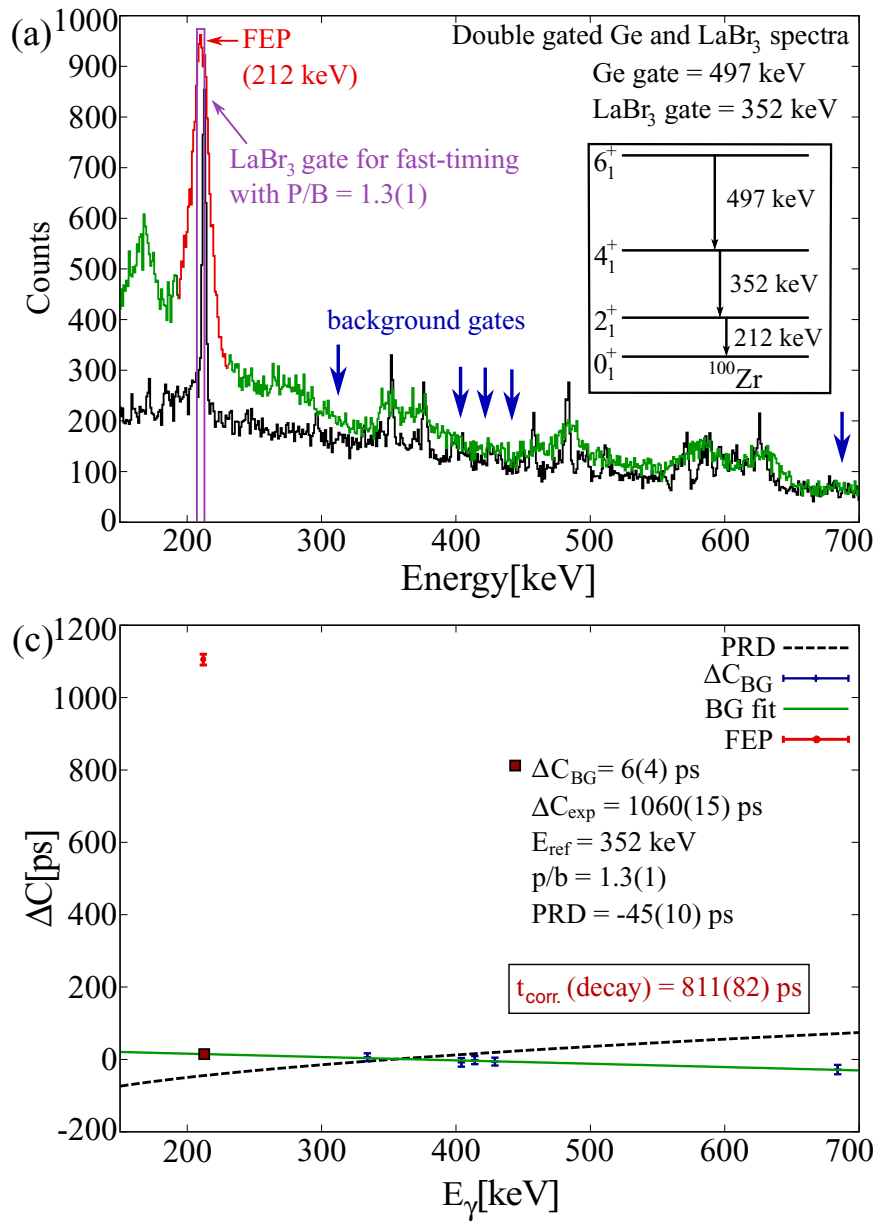

the decay $(621 \mathrm{keV})$ transition of the $4_{1}^{+}$level of ${ }^{98} \mathrm{Zr}$ are very close in energy and the energy resolution of the $\mathrm{LaBr}_{3}$ detectors for the EXILL-FATIMA array is not sufficient to distinguish unambiguously between these energies. Therefore, the summed lifetime of the $2_{1}^{+}$and $4_{1}^{+}$states of ${ }^{98} \mathrm{Zr}$ is measured and without a precise lifetime on the $2_{1}^{+}$state only an upper limit is obtained on the lifetime of the $4_{1}^{+}$state.

\section{2. ${ }^{100} \mathrm{Zr}$}

The lifetimes of the $2_{1}^{+}$and $4_{1}^{+}$states of ${ }^{100} \mathrm{Zr}$ were determined using GCDM as explained in Sec. II A. In addition, the slope method was also used to extract the $2_{1}^{+}$lifetime, as shown in Fig. 4. The spectra in Fig. 4(b), in contrast to those in Fig. 4(a), display two slope components, a fast (small bump at the beginning) and a slow one. Especially for lifetimes below $1 \mathrm{~ns}$, it is difficult to distinguish between the two slope components and select the time range in which only the slow slope component will be fitted. The different precision on the lifetime obtained using data from each of the fission targets is from a better peak-to-background ratio in the ${ }^{235} \mathrm{U}$ data. For comparison, if we try to apply Eq. (9) to $\triangle C_{\exp }$ values from Fig. 4 to extract the lifetime assuming no background, we obtain significantly different values $\left[603(11)\right.$ ps for ${ }^{235} \mathrm{U}$ target
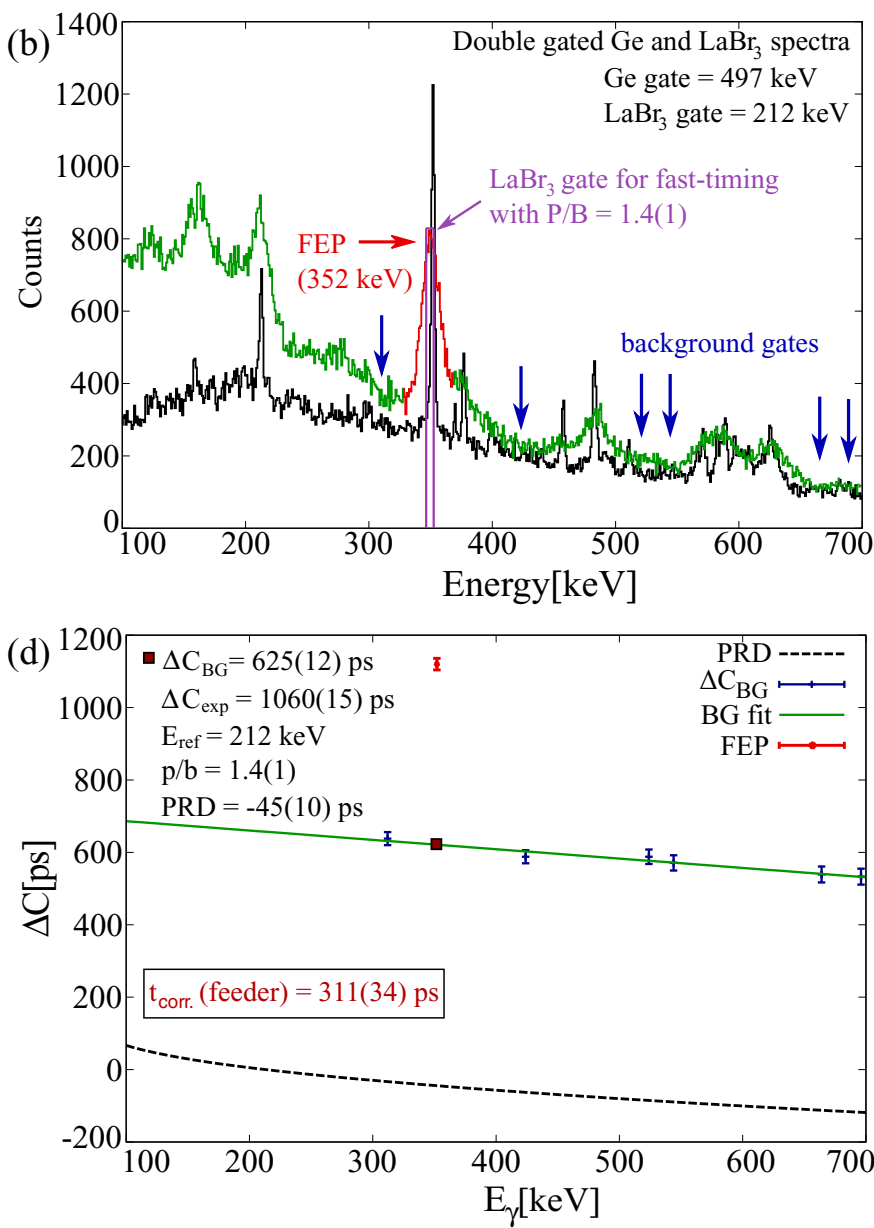

FIG. 5. Lifetime analysis for the $2_{1}^{+}$state in ${ }^{100} \mathrm{Zr}$. (a) and b) The double-gated Ge (shown in black) and $\mathrm{LaBr}_{3}$ [green (gray)] spectra. (c) and d) The Compton background correction procedure (see text for details). 


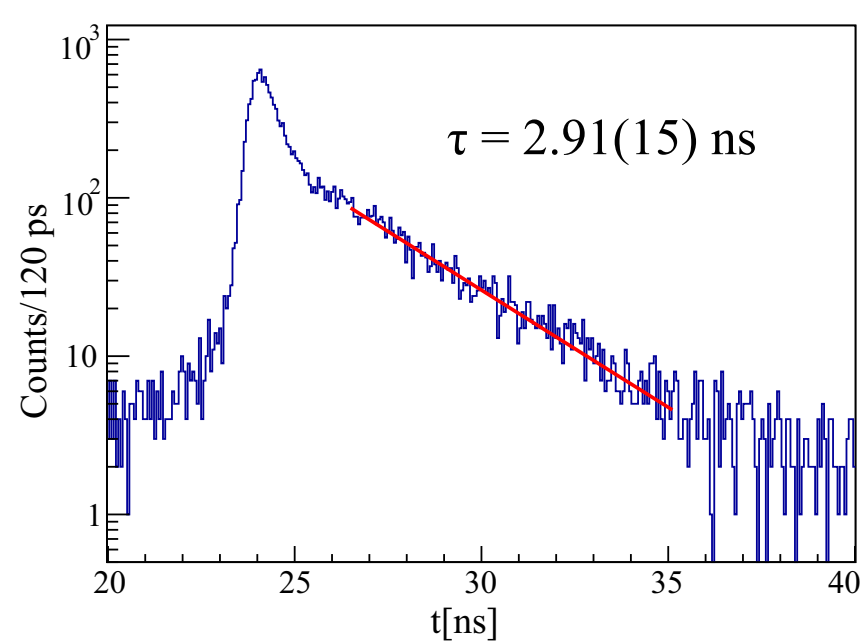

FIG. 6. Lifetime determination for the $2_{1}^{+}$state of ${ }^{102} \mathrm{Zr}$ using the ${ }^{241} \mathrm{Pu}$ target data. The independent antidelayed time spectrum resulting from the FEP events is inverted and aligned before being summed to the delayed time distribution. The slope was determined by fitting the data in the range from 25.5 to $35 \mathrm{~ns}$. and 509(9) ps for ${ }^{241} \mathrm{Pu}$ ] from those extracted using the slope method. This demonstrates that for lifetimes below $1 \mathrm{~ns}$ the correction for the Compton background should be performed, and consequently we further apply the GCDM with its reliable background correction procedure to the $2_{1}^{+}$state of ${ }^{100} \mathrm{Zr}$.

Figure 5 illustrates the complete GCDM procedure for the lifetime evaluation of the $2_{1}^{+}$state of ${ }^{100} \mathrm{Zr}$ with ${ }^{241} \mathrm{Pu}$ as a fission target. Figure 5(a) presents the double-gated $\left(\mathrm{Ge}+\mathrm{LaBr}_{3}\right)$ spectrum with $E_{\text {ref }}$ of $352 \mathrm{keV}$ (transition feeding the $2_{1}^{+}$ state) and FEP is the decay of the $2_{1}^{+}$state at $E=212 \mathrm{keV}$. A narrow energy gate of $6 \mathrm{keV}$ is applied on the FEP and the two centroids of independent delayed and antidelayed time distributions are calculated. The difference between these two time distribution centroids yields the $\triangle C_{\exp }$ value. The Compton background correction is performed by (1) finding the time distribution of the background through gating on a few background points in the vicinity of the FEP using the same channel width (six channels), (2) plotting the centroid difference of these background points against their respective energy, (3) fitting this dependence using a polynomial function, and (4) reading the $\triangle C_{\mathrm{BG}}$ at the position of the FEP from the thus
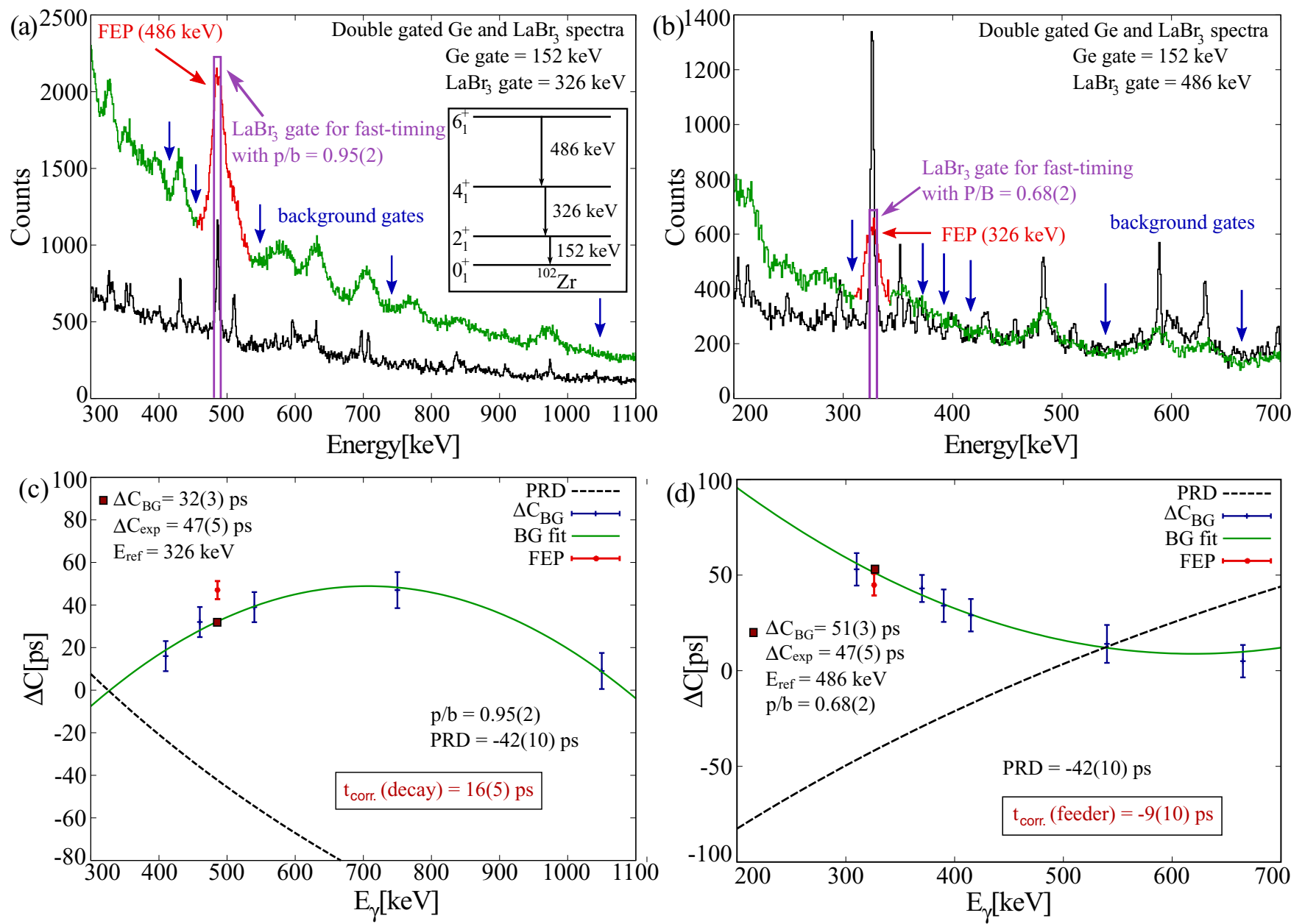

FIG. 7. Lifetime analysis for the $4_{1}^{+}$state in ${ }^{102} \mathrm{Zr}$. (a) and (b) Double-gated Ge (shown in black) and $\mathrm{LaBr}_{3}$ [green (light gray)] spectra. (c) and (d) The Compton background correction procedure (see text for details). 
obtained background curve [as shown in Fig. 5(c)]. The PRD correction is directly read from the PRD curve in Fig. 5(c). This curve is shifted with respect to the original plot (Fig. 2; details in Ref. [27]) to yield PRD equal to 0 at $E_{\text {ref }}$ of $212 \mathrm{keV}$.

The same procedure is repeated with the feeding and depopulating transitions interchanged ( $E_{\text {ref }}$ at $352 \mathrm{keV}$ and FEP at $212 \mathrm{keV}$ ). In this case, the background region is different and consequently different background gates are applied. It should be noted that the PRD curve as well as the Compton background correction curve in Fig. 5(d) are inverted with respect to those in Fig. 5(c) because the $E_{\text {ref }}$ is flipped from the transition feeding the state of interest to that depopulating it. Equations (7) and (9) are then applied to the values listed in Figs. 5(c) and 5(d) yielding the lifetime of the $2_{1}^{+}$level equal to $830(30) \mathrm{ps}$.

\section{3. ${ }^{102} \mathrm{Zr}$}

The lifetime of the $2_{1}^{+}$state of ${ }^{102} \mathrm{Zr}$ was determined using the slope method on data obtained from both targets. Figure 6 shows the time distribution observed with the ${ }^{241} \mathrm{Pu}$ target. The slow component of the slope is more prominent compared to that observed for ${ }^{100} \mathrm{Zr}$ (Fig. 4) because of the longer lifetime of the $2_{1}^{+}$level of ${ }^{102} \mathrm{Zr}$ and a relatively low background contribution to the peak. Consistent values were obtained for both targets: 2.91(15) ns for ${ }^{241} \mathrm{Pu}$ and 2.9(2) ns for ${ }^{235} \mathrm{U}$.

To determine the previously unknown lifetimes of the $4_{1}^{+}$ and $6_{1}^{+}$states, the GDCM was applied to the data collected using both ${ }^{235} \mathrm{U}$ and ${ }^{241} \mathrm{Pu}$ targets. The lifetime analysis for the $4_{1}^{+}$state of ${ }^{102} \mathrm{Zr}$ using the ${ }^{235} \mathrm{U}$ target data is presented in Figs. 7 and 8 . It follows the same procedure as for ${ }^{100} \mathrm{Zr}$ except that in this case the centroid difference related to the Compton background $\left(\triangle C_{\mathrm{BG}}\right)$ is fitted using a quadratic function. It is worth mentioning that for this level the background contribution was larger with respect to the FEP as was the $\triangle C_{\mathrm{BG}}$ correction. The parallel adjustment of the PRD curve is made as per Eq. (2) to cross the energy axis at the reference

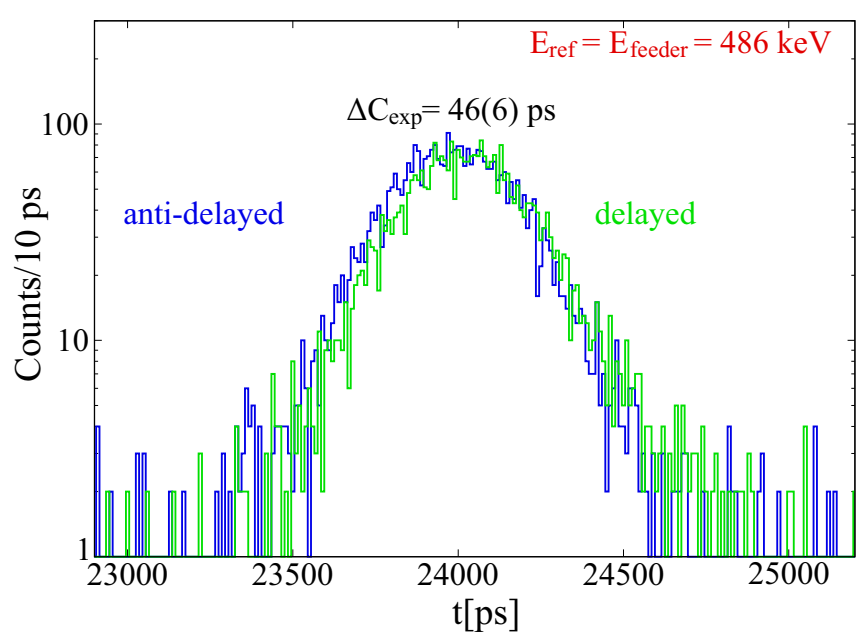

FIG. 8. Two independent time distributions (delayed and antidelayed) for the $4_{1}^{+}$state of ${ }^{102} \mathrm{Zr}$.

energy. It should be noted that the PRD curve in Figs. 7(c) and 5(c) does not change its shape for different reference energies and only a parallel shift is observed, which is related to the $\gamma-\gamma$ time walk of the corresponding energies. The lifetimes obtained for the $4_{1}^{+}$and $6_{1}^{+}$states with the ${ }^{241} \mathrm{Pu}$ target are influenced by the presence in the $\gamma$-ray spectra of the transitions in the complementary fission partner ${ }^{138} \mathrm{Xe}$, as explained in Sec. II B. This is, however, not the case for data collected with the ${ }^{235} \mathrm{U}$ fission target. A value of 46(7) ps was determined for the lifetime of the $4_{1}^{+}$state, using $t_{\text {corr }}($ feeder $)=$ $16(5)$ ps and $t_{\text {corr }}$ (decay) $=-9(10)$ ps. For the $6_{1}^{+}$state, an upper limit of 12 ps was obtained.

\section{RESULTS AND DISCUSSION}

The obtained lifetimes are presented in Table I. Only upper limits could be determined for the $2_{1}^{+}$and $4_{1}^{+}$states of ${ }^{98} \mathrm{Zr}$, and the $6_{1}^{+}$state of ${ }^{102} \mathrm{Zr}$, because of the low peak-to-background

TABLE I. Lifetimes of yrast states in ${ }^{98,100,102} \mathrm{Zr}$ extracted using fast-timing methods from the ${ }^{241} \mathrm{Pu}$ and ${ }^{235}$ U data from the EXILL-FATIMA campaign. All values are given in ps unless mentioned otherwise. The literature values are the most recent values from Evaluated and Unevaluated National Nuclear Data Center [7] with the original reference provided. All the lifetime results are quoted with $1 \sigma$ confidence limit.

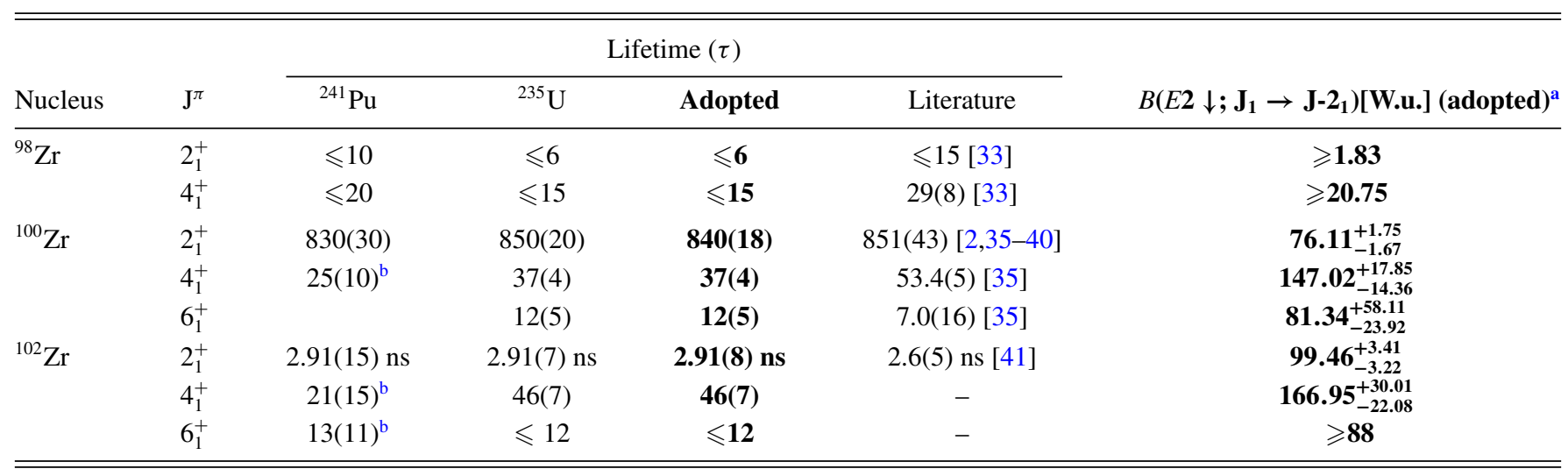

a 1 W.u equals to $26.84,27.57,28.31 \mathrm{e}^{2} \mathrm{fm}^{4}$ in ${ }^{98,100,102} \mathrm{Zr}$, respectively.

${ }^{\mathrm{b}}$ The lifetimes determined from the ${ }^{241} \mathrm{Pu}$ data are affected by the contamination from $\gamma$-ray transitions in the complementary fission partner as explained in Sec. II B. 

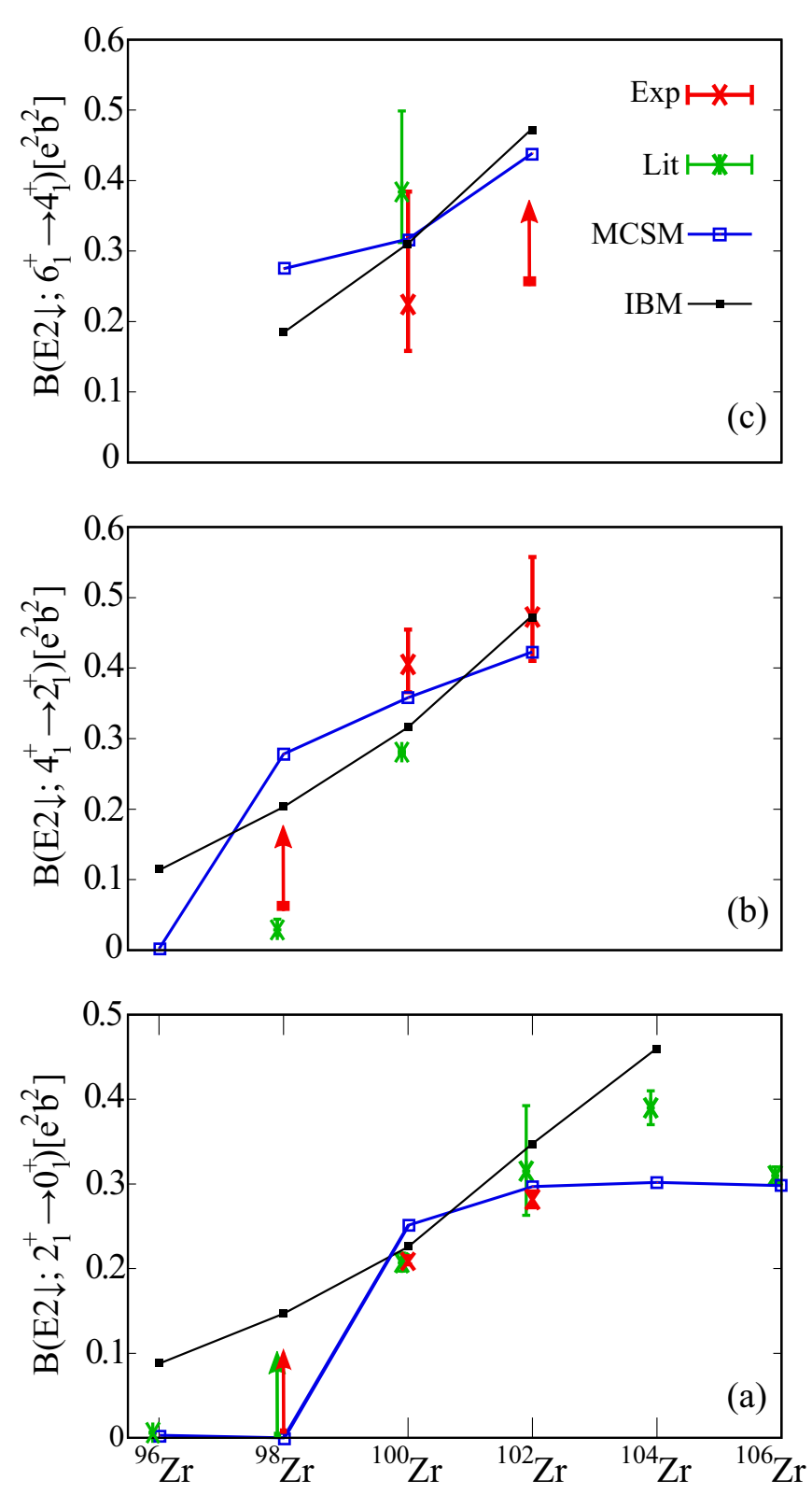

FIG. 9. Known $B\left(E 2 \downarrow ; 2_{1}^{+} \rightarrow 0_{1}^{+}\right), B\left(E 2 \downarrow ; 4_{1}^{+} \rightarrow 2_{1}^{+}\right)$, and $B(E 2$ $\downarrow ; 6_{1}^{+} \rightarrow 4_{1}^{+}$) values [a), (b), and (c), respectively] in ${ }^{98,100,102,104,106} \mathrm{Zr}$, compared with the IBM-1 [42] and MCSM [24] calculations. The $B(E 2) \downarrow$ values obtained in the present study (see Table I) are plotted in red and the literature values $[2,33-41,43,44]$ in green (light gray). All values are expressed in $e^{2} b^{2}$.

ratios and short lifetimes. We concluded from the analysis performed for the $2_{1}^{+}$state of ${ }^{100} \mathrm{Zr}$ that the slope method is sensitive to background for lifetimes below $1 \mathrm{~ns}$ whereas the GCDM gives consistent results for both targets even though the peak-to-background ratio was dramatically different. The lifetimes of the $4_{1}^{+}$and $6_{1}^{+}$states of ${ }^{100,102} \mathrm{Zr}$ measured using the ${ }^{241} \mathrm{Pu}$ fission target are significantly different from those obtained with ${ }^{235} \mathrm{U}$. This is related to the contamination of relevant $\gamma$-ray spectra by transitions in the complementary fission partner, as explained in Sec. II B.
Lifetimes in ${ }^{98} \mathrm{Zr}$ were previously measured in a $\beta-\gamma-\gamma$ experiment using the centroid shift method [33]. In ${ }^{100} \mathrm{Zr}$, lifetimes of the short-lived $4_{1}^{+}$and $6_{1}^{+}$states were measured using the Doppler profile method $[34,35]$ and the long-lived $2_{1}^{+}$ state using different techniques illustrated in Refs. [2,35-40]. Most of these values are in good agreement with the present results as shown in Table I.

The present experimental lifetime results are used to calculate the $B(E 2) \downarrow$ transition strengths that are compared with theoretical calculations using the interacting boson model (IBM-1) [42] and the Monte Carlo shell model (MCSM) [24], as shown in Fig. 9.

The IBM-1 calculations, described in detail in Ref. [42], used ${ }^{90} \mathrm{Zr}$ as the core. Good agreement with the present experimental results is found for ${ }^{100,102} \mathrm{Zr}$. Because only upper limits are currently known for the lifetimes in ${ }^{98} \mathrm{Zr}$, it is difficult to make firm conclusions on the evolution of transition probabilities from ${ }^{98} \mathrm{Zr}$ to ${ }^{100} \mathrm{Zr}$, which is predicted by the IBM-1 to be gradual. It should be noted that these calculations also predict a smooth change in the energy of the $2_{1}^{+}$state with increasing neutron number, contrary to the experimental observations (see Fig. 1). In contrast, the dramatic decrease of the $2_{1}^{+}$level energy when going from ${ }^{98} \mathrm{Zr}$ to ${ }^{100} \mathrm{Zr}$ was well reproduced by recent state-of-the-art MCSM calculation [24]. Unlike the conventional shell model calculations that are constrained by the size of the configuration space, the MCSM allows the calculation in large configuration spaces up to $3.7 \times 10^{23}$ two-body matrix elements. Our data on ${ }^{100,102} \mathrm{Zr}$ agree very well with the MCSM predictions, while the obtained upper limit on the $2_{1}^{+}$lifetime in ${ }^{98} \mathrm{Zr}$ does not permit the discrimination between the drastic phase transition at $N=60$ predicted by MCSM and a smooth onset of collectivity as per the IBM-1. Our lower limit on the $B\left(E 2 \downarrow ; 4_{1}^{+} \rightarrow 2_{1}^{+}\right)$value in ${ }^{98} \mathrm{Zr}$ is not in agreement with the literature value, but is consistent with both the MCSM and IBM- 1 calculations. The upper limit on the lifetime of the $6_{1}^{+}$state in ${ }^{102} \mathrm{Zr}$ does not allow for a meaningful comparison with either model predictions. Definite lifetimes in ${ }^{98} \mathrm{Zr}$ are required that will provide the final verdict on the phase transition in this region and also allow us to further investigate the phenomenon of shape coexistence.

\section{SUMMARY}

We studied lifetimes of yrast states in ${ }^{98,100,102} \mathrm{Zr}$ populated in neutron-induced fission of ${ }^{241} \mathrm{Pu}$ and ${ }^{235} \mathrm{U}$ using a combination of fast-timing $\mathrm{LaBr}_{3}(\mathrm{Ce})$ and EXOGAM clover detectors. The lifetimes were determined using the slope method, applicable for the lifetimes above approximately 1 ns, and the generalized centroid difference method for shorter lifetimes. The lifetime of the $4_{1}^{+}$state and an upper limit on the lifetime of the $6_{1}^{+}$state in ${ }^{102} \mathrm{Zr}$ were obtained for the first time. For other lifetimes determined in this study, good agreement was found with the literature values except for the limit on the $4_{1}^{+}$level of ${ }^{98} \mathrm{Zr}$ and the lifetime of the $4_{1}^{+}$level of ${ }^{100} \mathrm{Zr}$. The presently determined upper limits on the lifetimes in the ground-state band of ${ }^{98} \mathrm{Zr}$ do not permit conclusions on the possible shape phase transition in the $\mathrm{Zr}$ isotopic chain at $N=60$. 


\section{ACKNOWLEDGMENTS}

The author warmly acknowledges K. Moschner for the fruitful discussion during the data analysis of this work, and sincerely thanks P. Garrett for proofreading the earlier version of the manuscript. The EXILL\&FATIMA campaign would not have been possible without the support of several services at the ILL and the LPSC. We are grateful to the EXOGAM collaboration for the loan of the detectors, to GANIL for assistance during installation and dismantling, and to the FATIMA collaboration for the provision of $\mathrm{LaBr}_{3}(\mathrm{Ce})$ detectors and analog electronics. This work was supported by German BMBF under Grants No. 05P15PKFNA and No. 05P12PKNUF and by the UK Science and Technology facilities council and the UK National Measurement Office. Theoretical studies including MCSM calculations were supported in part by JSPS Grants-in-Aid for Scientific Research (Grant No. 23244049), in part by HPCI Strategic Program (Program No. hp150224), in part by MEXT and JICFuS and Priority Issue on Post-K computer (Elucidation of the fundamental laws and evolution of the universe) (Program No. hp160211), and by the CNS-RIKEN joint project for large-scale nuclear structure calculations.
[1] S. A. Johansson, Nucl. Phys. 64, 147 (1965).

[2] E. Cheifetz, R. C. Jared, S. G. Thompson, and J. B. Wilhelmy, Phys. Rev. Lett. 25, 38 (1970).

[3] D. Arseniev, A. Sobiczewski, and V. Soloviev, Nucl. Phys. A 139, 269 (1969).

[4] R. Sheline, I. Ragnarsson, and S. Nilsson, Phys. Lett. B 41, 115 (1972).

[5] S. Naimi, G. Audi, D. Beck, K. Blaum, C. Böhm et al., Phys. Rev. Lett. 105, 032502 (2010).

[6] F. Flavigny, P. Doornenbal, A. Obertelli, J.-P. Delaroche, M. Girod et al., Phys. Rev. Lett. 118, 242501 (2017).

[7] M. Chadwick, M. Herman, P. Obložinský et al., Nucl. Data Sheets 112, 2887 (2011); special Issue on ENDF/B-VII.1 Library.

[8] J. Dudouet, A. Lemasson, G. Duchêne, M. Rejmund, E. Clément, C. Michelagnoli, F. Didierjean et al., Phys. Rev. Lett. 118, 162501 (2017).

[9] M. Albers, N. Warr, K. Nomura, A. Blazhev, J. Jolie et al., Phys. Rev. Lett. 108, 062701 (2012); Errata: 109, 209904 (2012); 114, 189902 (2015).

[10] C. Sotty, M. Zielińska, G. Georgiev, D. L. Balabanski, A. E. Stuchbery, A. Blazhev et al., Phys. Rev. Lett. 115, 172501 (2015).

[11] T. W. Hagen, A. Görgen, W. Korten, L. Grente, M.-D. Salsac et al., Phys. Rev. C 95, 034302 (2017).

[12] K. Heyde and J. L. Wood, Rev. Mod. Phys. 83, 1467 (2011).

[13] E. Clément, M. Zielińska, A. Görgen, W. Korten, S. Péru et al., Phys. Rev. Lett. 116, 022701 (2016).

[14] E. Clément, M. Zielińska et al., Phys. Rev. C 94, 054326 (2016).

[15] J.-M. Régis, J. Jolie, N. Saed-Samii, N. Warr et al., Phys. Rev. C 95, 054319 (2017); 95, 069902(E) (2017).

[16] F. Schussler, J. Pinston, E. Monnand, A. Moussa, G. Jung et al., Nucl. Phys. A 339, 415 (1980).

[17] J. Park, A. B. Garnsworthy, R. Krücken, C. Andreoiu, G. C. Ball, P. C. Bender, A. Chester, A. Close, P. Finlay, P. E. Garrett et al., Phys. Rev. C 93, 014315 (2016).

[18] F. Wohn, H. Mach, M. Moszyński, R. Gill, and R. Casten, Nucl. Phys. A 507, 141 (1990).

[19] H. Mach, M. Moszyński, R. L. Gill, G. Molnár, F. K. Wohn, J. A. Winger, and J. C. Hill, Phys. Rev. C 41, 350 (1990).

[20] C. Y. Wu, H. Hua, and D. Cline, Phys. Rev. C 68, 034322 (2003).

[21] E. Clément, A. Görgen, W. Korten, E. Bouchez, A. Chatillon, J.-P. Delaroche et al., Phys. Rev. C 75, 054313 (2007).
[22] N. Bree, K. Wrzosek-Lipska, A. Petts, A. Andreyev, B. Bastin et al., Phys. Rev. Lett. 112, 162701 (2014).

[23] K. Sieja, F. Nowacki, K. Langanke, and G. Martínez-Pinedo, Phys. Rev. C 79, 064310 (2009).

[24] T. Togashi, Y. Tsunoda, T. Otsuka, and N. Shimizu, Phys. Rev. Lett. 117, 172502 (2016).

[25] T. Otsuka and Y. Tsunoda, J. Phys. G: Nucl. Part. Phys. 43, 024009 (2016).

[26] H. Abele, D. Dubbers, H. Häse, M. Klein, A. Knöpfler, M. Kreuz, T. Lauer, B. Märkisch et al., Nucl. Instrum. Methods Phys. Res. Sect. A 562, 407 (2006).

[27] J.-M. Régis, G. Simpson, A. Blanc, G. de France, M. Jentschel, U. Köster, P. Mutti et al., Nucl. Instrum. Methods Phys. Res. Sect. A 763, 210 (2014).

[28] W. Urban, M. Jentschel, B. Märkisch, T. Materna, C. Bernards, C. Drescher, C. Fransen, J. Jolie, U. Köster, P. Mutti, T. RzacaUrban, and G. S. Simpson, J. Instrum. 8, P03014 (2013).

[29] P. Mutti, A. Blanc, G. de France, M. Jentschel, U. Köster, E. R. Martinez, G. Simpson, T. Soldner, C. A. Ur, and W. Urban, in Proceedings of the 3rd International Conference on Advancements in Nuclear Instrumentation Measurement Methods and their Applications (ANIMMA), Marseille, France (IEEE, Piscataway, NJ, 2013), pp. 1-6.

[30] N. Saed-Samii, Diploma thesis (unpublished), Institute of Nuclear Physics, Cologne, 2015.

[31] J.-M. Régis, G. Pascovici, J. Jolie, and M. Rudigier, Nucl. Instrum. Methods Phys. Res. Sect. A 622, 83 (2010).

[32] J.-M. Régis, H. Mach, G. Simpson, J. Jolie, G. Pascovici, N. Saed-Samii, N. Warr et al., Nucl. Instrum. Methods Phys. Res. Sect. A 726, 191 (2013).

[33] L. Bettermann, J.-M. Régis, T. Materna, J. Jolie, U. Köster, K. Moschner, and D. Radeck, Phys. Rev. C 82, 044310 (2010).

[34] A. Smith, D. Patel, G. Simpson, R. Wall, J. Smith, O. Onakanmi, I. Ahmad, J. Greene et al., Phys. Lett. B 591, 55 (2004).

[35] A. G. Smith, R. M. Wall, D. Patel, G. S. Simpson, D. M. Cullen, J. L. Durell, S. J. Freeman et al., J. Phys. G: Nucl. Part. Phys. 28, 2307 (2002).

[36] H. Mach, M. Moszynński, R. Gill, F. Wohn, J. Winger, J. C. Hill, G. Molnár, and K. Sistemich, Phys. Lett. B 230, 21 (1989).

[37] H. Ohm, M. Liang, G. Molnár, and K. Sistemich, Z. Phys. A At. Nucl. 334, 519 (1989).

[38] G. Lhersonneau, H. Gabelmann, N. Kaffrell, K.-L. Kratz, B. Pfeiffer, and the ISOLDE Collaboration, Z. Phys. A At. Nucl. 332, 243 (1989). 
[39] E. Cheifetz, H. A. Selic, A. Wolf, R. Chechik, and J. B. Wilhelmy, Proceedings of the Conference on Nuclear Spectroscopy of Fission Products (IAEA, Vienna, Austria, 1979), Vol. 11.

[40] R. C. Jared, H. Nifenecker, and S. G. Thompson, in Nuclear Physics and Radiation Physics, Proceedings of the Symposium on the Physics and Chemistry of Fission on Atomic Energy in Food and Agriculture, Rochester, NY, 1973 (IAEA, Vienna, Austria, 1974), Vol. 5, pp. 211-219.
[41] S. Raman, C. Nestor, and P. Tikkanen, At. Data Nucl. Data Tables 78, 1 (2001).

[42] J. E. García-Ramos, K. Heyde, R. Fossion, V. Hellemans, and S. De Baerdemacker, Eur. Phys. J. A 26, 221 (2005).

[43] F. Browne, A. Bruce, T. Sumikama, I. Nishizuka, S. Nishimura, P. Doornenbal et al., Phys. Lett. B 750, 448 (2015).

[44] G. Kumbartzki, N. Benczer-Koller, J. Holden, G. Jakob, T. Mertzimekis et al., Phys. Lett. B 562, 193 (2003). 